\title{
Philosophiques
}

\section{Rationalité scientifique et rationalité du mythe}

\section{Henri Atlan}

Volume 22, numéro 2, automne 1995

URI : https://id.erudit.org/iderudit/027332ar

DOI : https://doi.org/10.7202/027332ar

Aller au sommaire du numéro

Éditeur(s)

Société de philosophie du Québec

ISSN

0316-2923 (imprimé)

1492-1391 (numérique)

Découvrir la revue

Citer cet article

Atlan, H. (1995). Rationalité scientifique et rationalité du mythe. Philosophiques, 22(2), 265-280. https://doi.org/10.7202/027332ar d'utilisation que vous pouvez consulter en ligne.

https://apropos.erudit.org/fr/usagers/politique-dutilisation/ 


\title{
$\begin{array}{lllllllllllll}\text { I } & \text { N } & \text { I } & \text { E } & R & \text { V } & \text { E } & \text { N } & \text { I } & \text { I } & \text { O } & \text { N } & \text { S }\end{array}$
}

\section{RATIONALIIÉ SCIENTIFIQUE ET RATIONALIIÉ DU MYTHE}

\author{
par \\ Henri Atlan
}

Depuis trois siècles, les sciences de la nature sont devenues de plus en plus efficaces pour permettre aux hommes de se prémunir contre les aléas, les douleurs et les cruautés de l'existence. Mais cette maîtrise de plus en plus grande de l'espace et du temps n'est acquise qu'au prix d'une dépersonnalisation de plus en plus grande. Contrairement au monde mythique de l'animisme et de la magie, celui des sciences et des techniques n'est plus animé, ne parle plus qu'un langage de signes abstraits, beaucoup plus efficace que le langage des anciennes sciences initiatiques telles que l'alchimie, les tarots ou le I King en Chine.

Le langage de ces anciennes sciences était lui aussi abstrait et parfois très élaboré, mais en même temps il parlait directement des expériences de la vie des hommes puisqu'il supposait que l'Univers tout entier était un organisme vivant, animé par l'Âme du monde. Cette science de la Renaissance qu'on appelait alors la magie naturelle, celle des grands pionniers de la science moderne que furent par exemple Giordano Bruno, Paracelse, Pic de La Mirandole, était à la fois un savoir sur la nature, une théologie, une religion et une morale ou chaque chose dans l'Univers était à sa place, avait un sens rapporté à celui du cosmos ; il s'agissait aussi d'un ensemble de techniques de divination, d'action et de transformation de la nature par la recherche d'une maîtrise des forces cachées de l'Univers.

Mais aujourd'hui, tout cela est fini car la maîtrise de la matière et de l'énergie, et aussi du monde vivant, est acquise par une science et des techniques qui ont du se dissocier de la religion et des traditions initiatiques, et qui ont dû, pour être efficaces, respecter scrupuleusement un principe d'objectivité où les croyances individuelles, la sensibilité et la subjectivité du 
vécu de chacun, et même la question des valeurs morales ont été mises entre parenthèses. Même les sciences du vivant, et c'est cela qui est nouveau et peut-être le plus difficile à accepter pour certains, sont dévitalisées et complètent la dépersonnalisation de notre représentation du monde, puisque la biologie n'est devenue vraiment efficace qu'en devenant physico-chimique, moléculaire, et en se débarrassant du vitalisme. Cela veut dire que même l'organisation et le fonctionnement des organismes vivants sont compris et représentés aujourd'hui comme des éléments d'un monde tout entier animé. De cette situation nouvelle, il résulte pour nous qu'il existe un champ d'expérience où nous découvrons une réalité à laquelle la méthode scientifique n'est pas adaptée. C'est la réalité que nous pouvons atteindre à travers le mythe, dont l'art est une forme désacralisée.

Comme à la réalité que nous appelons objective, qui est l'objet des sciences, nous avons accès à cette réalité-là à la fois par nos sens et par notre raison.

Nous y accédons par nos sens à travers ce qu'on appelait traditionnellement l'imagination, c'est-à-dire notre perception d'images qui nous font voir, ou entendre, ou sentir, des choses de façon différente, modifiée par rapport à notre perception habituelle. La forme la plus courante en est le rêve, mais elle comprend aussi les états modifiés de conscience, spontanés ou produits par certains exercices et par des substances hallucinogènes qui ont joué - et jouent encore dans certaines tribus - un rôle mythogène important.

Des études sur les aborigènes d'Australie ont montré qu'il existe une continuité de fait entre leurs rêves, les mythes organisateurs de leur société et de leur territoire, et les rites par lesquels ces mythes sont exprimés et pérennisés ${ }^{1}$.

Mais nous accédons à cette réalité aussi par la raison, car il existe une rationalité du mythe. Pour s'en rendre compte, il est intéressant, dans une démarche généalogique, de situer la rationalité scientifique dans le temps par rapport à une rationalité préscientifique d'où elle est issue, celle de la magie naturelle de la Renaissance notamment. Mais de plus, dans une démarche comparative ou plus exactement, intercritique, il est important de situer la rationalité scientifique par rapport à une rationalité extrascientifique telle que celle du mythe. Cette intercritique consiste à analyser pour les faire jouer les unes contre les autres, non pas les ressemblances et les analogies, mais les différences entre l'activité scientifique et celle du mythe et du rite ${ }^{2}$. Très rapidement ici, nous pouvons indiquer successivement en quoi ces différences portent sur la finalité, sur la méthode, sur les objets et sur le langage de ces deux formes d'activités.

La finalité des sciences, c'est surtout la maîtrise de la nature grâce au pouvoir prédictif de théories et de modèles qui peuvent être de plus en plus

1. B. Glowczewski, Du rêve à la loi chez les Aborigènes, PUF, Paris, 1991.

2. Henri Atlan, À tort et d̀ raison, Seuil, Paris, 1986. 
abstraits, mais ils sont acceptés comme valides tant qu'ils permettent cette maîtrise au moins partielle et cette reconstruction de la nature. La finalité des mythes est l'organisation des expériences humaines du vécu individuel et social, de ce qu'on appelle la vie intérieure et la vie en société ; expériences de la naissance et de la mort, de l'alimentation et de la sexualité, de la famille, de la tribu et de sa langue, tout cela en continuité avec une organisation globale du cosmos assignant sa place et son sens à chacune de ces expériences.

La méthode aussi est différente : de bas en haut dans les sciences, par abstractions et généralisations progressives et forcément partielles à partir d'observations et d'expériences limitées. Celles-ci sont circonscrites à tel ou tel domaine qui constitue une discipline scientifique particulière dont l'objet est défini en général par ses techniques d'observation et de mesure. Au contraire, méthode de haut en bas dans l'explication par le mythe, à partir d'une vision globale et organisée d'un tout de l'Univers qui sert de grille pour percevoir, interpréter et donner un sens à tout ce qui peut arriver.

En ce qui concerne les objets, ceux des sciences sont des choses et des processus observés de façon collective et généralisable, indépendante autant que possible des événements de la vie et de la subjectivité des individus qui les observent. L'objectivité scientifique implique la séparation entre le sujet comme subjectivité et l'objet, même quand la situation d'observation interfere avec l'observé, et qu' on doit tenir compte comme on dit du point de vue de l'observation. Au contraire, dans le mythe, la vie intérieure communique directement avec la réalité extérieure, comme dans le rêve et dans la magie. L'objet ne peut pas être séparé du sujet, car celui-ci investit tous les objets qu'il perçoit en même temps qu'il en est affecté.

Enfin le langage est différent : aussi transparent et univoque que possible en ce qui concerne le langage scientifique, avec les mathématiques comme idéal de rigueur et de précision ; au contraire langage métaphorique du mythe, interprétable et générateur de sens à l'infini.

Mais cela n'est pas si simple. Le langage mathématique, ayant atteint une précision syntaxique maximum, se trouve dépouillé de contenu sémantique propre, de référence propre à autre chose que lui-même, et de ce fait, il peut donner lieu à des interprétations multiples quant à la signification de ses projections sur le monde physique. Inversement, le langage du mythe peut se formaliser. C'est en tous cas ce qui s'est passé dans l'histoire de quelques grandes traditions, où l'expérience mystique, pour être transmise, s'est coulée dans le cadre d'un langage formel. Celuici s'est exprimé dans des formules dont l'enchaînement obéit à une logique qui lui est propre, mais nous est parfaitement accessible. C'est le cas notamment en Occident des traditions mystiques de la Kabbale et de l'alchimie, et aussi de l'Hermétisme de la Renaissance, c'est-à-dire d'une ancienne gnose grecque. Ces savoirs traditionnels ont conduit à ce qu'on a appelé alors la magie naturelle dont on connaît de plus en 
plus le rôle aux origines de ce qui allait devenir la science moderne au XVII siecle $^{3}$. Et dans cette histoire, la rupture de la science moderne d'avec la magie naturelle, science de la Renaissance, est aussi importante que la continuité. Mais la trace de ces origines reste encore, jusqu'aujourd'hui, source de confusions.

Il arrive de plus qu'une théorie scientifique joue le rôle d'un mythe d'origine comme les théories cosmogoniques sur l'origine de l'univers, ou la théorie de l'évolution sur l'origine de l'homme. Et inversement, que des récits mythiques par ailleurs très riches soient utilisés au premier degré, de façon dogmatique. L'exemple le plus spectaculaire est celui du récit biblique de la Genèse que les créationnistes voudraient considérer comme une théorie scientifique en compétition avec la théorie de l'évolution.

Et comme la rationalité scientifique a évolué et a changé de forme au cours de son histoire, on risque toujours de tomber dans l'un de deux pièges symétriques, qui reviennent tous deux à ne pas concevoir que se sont constituées différentes façons de se servir de la raison, non réductibles les unes aux autres. La raison est la même mais son usage est différent, c'est-à-dire le type de relation qu'on établit entre un discours rationnel et son objet. Autrement dit, la réalité que ce discours est censé représenter et expliquer. Un piège consiste à croire que seule la connaissance scientifique est rationnelle et que tout le reste est soit illusion, soit confusion de l'irrationnel. Comme disait Russell, «il n'y a de vrai que le scientifique », qui impliquait « il n'y a de vrai que le rationnel » et «il n'y a de rationnel que le scientifique ». L'autre piège consiste à croire que la raison scientifique s'est transformée au $\mathrm{XX}^{\mathrm{e}}$ siècle par rapport à ce qu'elle était jusque là, et qu'elle s'est élargie jusqu'à retrouver les enseignements des grandes traditions mystiques.

Ce sont ces deux pièges que nous devons éviter tout en sachant que la tentation revient sans cesse de reconstituer une grande synthèse unificatrice, soit sous la forme d'une idéologie philosophique, et il semblerait que ce qu'on appelle l'épistémologie évolutionniste aurait tendance à jouer ce rôle, soit sous la forme d'une synthèse scientificomystique utilisant un jargon pseudoscientifique moderne, souvent néovitalisme et panpsychique voyant de la vie et de la conscience partout. Ce jargon sert à habiller les anciennes sciences et à les mettre au goût du jour, par exemple dans la nouvelle alchimie, ou l'astrologie dite scientifique, ou la culture du New Age. Enfin, la tentation de la grande synthèse s'exprime parfois dans une idéologie politique qu'on observe dans certaines tendances du mouvement écologistes dites non-anthropocentriques, comme la «deep ecology», ou le sens de l'évolution tend à remplacer le sens de l'histoire ; ou la protection de la Vie et de la Nature comme abstractions pourraient prendre la place de la lutte des classes et de la dictature du prolétariat pour justifier les inégalités et les oppressions

3. F. Ayate, Giordano Bruno and the Hermetic tradition, Univ. Chicago Press, 1964. 
naturelles et «vivantes » d'individus humains réels dans le concret de leur existence.

Or, une des raisons, sur le plan théorique, pour lesquelles ces deux pièges sont difficiles à éviter, est que cette séparation entre des rationalités différentes, opérant chacune dans des domaines de légitimité différents, est relativement nouvelle. Elle se heurte à une façon de penser unificatrice dominante depuis des siècles, sinon des millénaires.

Pour s'en rendre compte, le mieux est de retracer brièvement l'histoire de ce qu'on a appelé la révolution scientifique. Plus précisément, le passé de la science, vu d'aujourd'hui, est marqué par deux révolutions. L'une est justement ce qu'on a appelé la révolution scientifique au XVII ${ }^{e}$ siècle, qui fut une révolution de la physique. La deuxième est survenue dans notre siècle : c'est la révolution biologique. En ce qui concerne la première, l'utilisation du calcul dans l'intégration et la prédiction de résultats de mesures expérimentales en est la marque la plus évidente. Une de ses conséquences les plus importantes fut l'élimination définitive des causes finales du domaine de légitimité d'une explication scientifique. La physique des corps célestes et des autres corps pouvait ainsi se débarrasser de la finalité des entéléchies et des âmes. Le principe de raison suffisante imposait de se restreindre aux causes efficientes et renvoyait à un animisme primitif l'invocation de finalités intentionnelles dans la gestion des lois physiques. Autrement dit, la révolution scientifique du XVII ${ }^{\mathrm{e}}$ siècle, ce n'est pas la raison qui vient remplacer l'irrationnel, c'est un nouvel usage de la raison, d'où les causes finales sont chassées, qui s'impose à cause des succès de la mécanique.

Mais cette rupture ne fut pas d'emblée totale. Elle ne fut en fait radicale qu'en physique, tandis que trois siècles étaient nécessaires pour que la finalité, l'intention, l'âme soient aussi chassées des sciences du vivant.

Tout cela peut apparaître comme une controverse abstraite de philosophe logicien « des causes finales existent-elles ou non dans la nature ? » Mais, en fait, il y a là un enjeu capital concernant le sens de notre existence, dont nous mesurons aujourd'hui les conséquences.

Pour s'en rendre compte, il suffit de rappeler par quelques traits, de quoi étaient faites les philosophies de la nature à la Renaissance d'où est sortie la science classique, en rupture bien sûr, mais longtemps marquée par ses origines.

Au XVII ${ }^{\mathrm{e}}$ siècle, nous avons quitté la scolastique, et des pionniers comme Kepler et Newton sont encore très profondément nourris de ce qui nous apparaît aujourd'hui comme une rationalité mystique, celle de l'alchimie et de la Kabbale. Avant eux, qui créent véritablement la rupture et instituent la nouvelle raison, un Paracelse, médecin et astrologue, représente assez bien cette intrication extraordinaire d'intuitions qui annoncent la médecine expérimentale du XIX ${ }^{\mathrm{e}}$ siècle, et de pensée magique et alchimique, rationalisée dans une philosophie de la nature dont le pouvoir explicatif, à défaut d'efficacité technique, est des plus séduisants. 
C'est ainsi par exemple qu'on y trouve affirmé le primat de l'expérience sur la révélation livresque ; mais c'est pour mieux justifier des pratiques alchimiques et astrologiques dont «tout le monde » était censé pouvoir faire l'expérience de la réalité. Un autre exemple est celui de l'action magique de l'imagination par l'image qui permet d'expliquer les actions à distance et apparaît ainsi comme manifestation d'une toute-puissance humaine caractéristique de la magie dans un monde enchanté, peuplé d'elfes, d'anges, etc. Cette action de l'image, "l'imaginal " des mystiques d'après H. Corbin, sera reprise, modernisée en « imago » par C. G. Jung et les nouveaux alchimistes pseudo scientifiques du $\mathrm{XX}^{\mathrm{e}}$ siècle.

Ces exemples, quand on y regarde de plus près, montrent à l'évidence que ce n'est pas l'irrationalité qui caractérise cette pensée, ni même la fuite dans les mystères et le refus du verdict de l'expérience. Bien au contraire. Seulement, les expériences et les faits étaient reçus à partir de certains à priori, situés à l'intérieur d'un cadre de pensée qui était celui de la Renaissance, et plus exactement des hommes éclairés, chercheurs et philosophes de la Renaissance. Ce cadre de pensée nous apparaît aujourd' hui - à juste titre - comme antiscientifique, mythologique, mais il était alors celui qui a ouvert la route à la pensée scientifique, comme l'alchimie par rapport à la chimie. Il s'agit d'une pensée animiste et magique, mais qui se veut rationnelle et critique. Elle differe de la pensée scientifique qui lui succédera en ce qu'il s'agit d'un panvitalisme ou l'expérience première la plus évidente, qui sert de paradigme et de point de départ à toute pensée, n'est pas celle de la mécanique, du pendule ou d'autres mouvements de corps pesants, mais celle de l'évolution et du développement des êtres vivants dont nous avons, dans notre vie et de l'intérieur, une expérience immédiate. Il en découle toute une série de conséquences qui la rendent incompréhensible si on veut l'entendre dans le cadre conceptuel de la science d'aujourd'hui. Comme le dit A. Koyre à propos de Paracelse : «La dissolution de la science médiévale, avait chez lui, plus que chez quiconque de ses contemporains, provoque une renaissance et une revivification des superstitions les plus primitives ${ }^{4} \gg$. Au contraire de ses prédécesseurs scolastiques, ce n'est pas dans les livres de la tradition qu'il veut chercher à comprendre, mais « dans le monde, dans la réalité, dans la vie et dans la nature qu'il veut chercher ses enseignements et ses maitres ${ }^{5} »$. Mais cette nature, pour lui comme pour les philosophes de la Renaissance, est avant tout vivante et animée comme nous le sentons et l'expérimentons de nous-mêmes. Et «ce qui est le plus caractéristique pour toute cette école de pensée, c'est qu'elle est tout entière animée de la croyance que les processus du monde extérieur, du monde physique, ne font que répéter et symboliser ceux de

4. A. Koyré. Mystiques, Spirituels, Alchimistes du XVI siècle allemand, Paris, Gallimard, 1971, p. 180.

5. Ibid., p. 81 . 
l'âme $^{6}$ ". Les difficultés auxquelles elle se heurte sont celles de toute pensée du vivant qui bute sur la question, encore aujourd'hui d'actualité, de la « réalité » du possible, du mode d'être du potentiel.

Ces difficultés étaient surmontées par une espèce de fuite en avant que permettait la pensée mystique ou la notion de l'imagination, « intermédiaire magique entre la pensée et l'être ${ }^{7}$ ", conduisait à une crédulité sans bornes ou l'expérience servait de fondement à toutes les superstitions, car rien n'était posé comme impossible à cette nature vivante. On sait, au contraire, que la science moderne a véritablement commencé, la chimie et la physique, après l'alchimie, avec l'établissement de principes d'impossibilité : conservation de la masse pour la chimie, impossibilité du mouvement perpétuel pour la physique. Ce sont ces principes de limitation, auxquels s'en ajouteront d'autres plus tard, qui, par les possibilités de quantifications et de calcul qu'ils permettaient de développer, se sont révélés les plus féconds dans l'entreprise de transformation et de maîtrise de la matière. Quitte à refouler le rôle de l'imagination au domaine de la vie intérieure et d'un organicisme métaphysique qui caractérisera la philosophie du romantisme.

En particulier, les difficultés liées à l'existence potentielle dans le germe vivant, étaient résolues par l'invocation de causes finales. Comme ces causes finales étaient alors directement rapportées à la volonté de Dieu, rien n'était impossible à cette volonté divine toute puissante. C'est justement cette crédulité qui fut dénoncée par Descartes, et surtout par Spinoza qui, de façon radicale, rejeta purement et simplement toute finalité dans la nature au domaine de l'illusion anthropomorphique. Pour lui, c'est dans la croyance en un certain type de finalité ou d'intentionnalité quel qu'il soit, dirigeant et orientant la nature, que se trouve le principal obstacle à la connaissance scientifique, ce qu'il appelle « l'asile de l'ignorance », lieu ou l'ignorance peut se réfugier et s'abriter derrière l'idée des fins impénétrables de Dieu.

C'est en cela que Spinoza était considérablement en avance sur son temps - et que son Éthique retrouve une actualité insoupçonnée - puisque c'est seulement aujourd'hui, avec la deuxième révolution, la révolution biologique, que nous pouvons apprécier à quel point l'idée de fins de la nature est incompatible avec la démarche scientifique. Même l'idée de finalité interne de Kant qui pouvait encore être soutenue tant que le vitalisme pouvait servir de moteur et de théorie dominante en biologie, apparaît aujourd'hui pour ce qu'elle était ; à savoir, le fondement d'une théologie morale dont le seul rapport possible aux sciences de la nature et à la philosophie critique, ne subsistait que grâce au vitalisme de l'époque, c'est-à-dire à la reconnaissance de cette-finalité interne. Celle-ci, caractéristique de la vie, aurait distingué radicalement les êtres vivants des mécanismes physicochimiques et empêché à tout jamais que nous puissions les connâttre suffisamment d'après de simples

6. Ibid., p. 114.

7. Ibid., p. 99. 
principes mécaniques, sans faire appel à l'existence d'une finalité intentionnelle, intelligente, à l'œuvre dans la nature.

Kant refusait la possibilité même d'une révolution mécanique en biologie analogue à celle de Newton en physique. Il écrivait en 1790 qu'il est absurde pour des hommes « d'espérer qu'il surgira un jour quelque Newton qui pourrait faire comprendre ne serait-ce que la production d'un brin d'herbe d'après les lois naturelles qu' aucune intention n'a ordonnée ${ }^{8} »$. Le XVIII ${ }^{\mathrm{e}}$ et le $\mathrm{XIX}^{\mathrm{e}}$ siècles et la moitié du $\mathrm{XX}^{\mathrm{e}}$ ont vécu sur cette dichotomie entre sciences physiques et sciences de la vie. Ce furent les beaux jours du vitalisme scientifique qui inspira de grandes philosophies de la vie, soit religieuses, soit romantiques et athées comme celles d'un Schopenhauer, d'un Nietzsche.

Mais la situation était instable, car depuis que les âmes avaient été chassées des astres, ce n'était plus la Vie qui servait de modèle à la physique, mais au contraire les modèles physico-chimiques qui pénétraient la biologie. Ainsi, les causes finales, circonscrites au domaine du vivant, étaient devenues un problème, le problème de la biologie. Plus exactement, elles sont restées comme une écharde, une question permanente, sans cesse refoulée et rouverte, au coeur de la biologie ; une contradiction, d'ailleurs féconde, entre une inspiration vitaliste apparemment irréductible au physico-chimique, et l'institution de la biologie en science expérimentale dont les modeles sont ceux de la physicochimie et de la mécanique. C'est ainsi que la question de la finalité et de l'intention dont nous faisons l'expérience intérieure dans notre capacité de faire des projets, et sur laquelle d'ailleurs repose notre vie sociale, morale et juridique, a hanté la biologie pendant à peu près trois siècles; depuis qu'elle a tenté de s'instituer en science de l'animé en prenant ses modèles dans les sciences de l'inanimé.

Or, cette question a été réglée aujourd'hui par la biologie physicochimique et moléculaire qui a réussi, à son tour, à éliminer les âmes du vivant. Mais elle rebondit de plus belle depuis que la psyché humaine est devenue maintenant objet de science ; en particulier depuis qu' on s'interroge à son sujet sur les structures d'un psychisme inconscient. Aucune interrogation sur l'inconscient ne peut éviter aujourd'hui la question de ses finalités et intentionnalités. Car la dynamique de l'inconscient peut être vue en prenant comme modèle soit la machine, soit nous-mêmes vus à travers nos expériences de sujets.

C'est ainsi qu'on parle :

- soit de machine à désirer, machine à parler, machine à fabriquer du sens pour désigner un inconscient qui apparaît comme une machine spécifique, mais machine tout de même ;

- soit de double d'un sujet, souffrant, parlant, sentant et pensant, double d'un patient donc, au sens propre et figuré, avec sa stratégie, ses ruses,

8. Emmanuel Kant. Critique de la faculté de juger, trad. A. Philonenko, Paris, Vrin, 1986, p. 214. 
ses discours. Dans ce cas, souvent, tout se passe comme si on lui attribuait les propriétés d'un sujet conscient, certes caché derrière la conscience, mais fonctionnant comme un double.

Quoiqu'il en soit, pour la biologie d'aujourd'hui, la question est réglée : la seule réalité concevable est mécanique à la façon d'une machine auto-organisatrice qui fabrique de façon automatique et sans intention ce qui semble être un programme.

Autrement dit, il n'existe pas dans la nature d'intentions et de projets autres que ceux dont nous faisons l'expérience, de façon subjective, quand nous délibérons avec nous-mêmes, décidons d'un but à atteindre et fonctionnons alors avec-le-sentiment d'être guidés par la fin que nous poursuivons.

Prendre conscience ainsi, contrairement aux prédictions de Kant, du caractère mécanique, sans projet, de la nature du vivant, a des conséquences qu'on peut juger destructrices, mais qui n'ont pas que des inconvénients. Cela détruit en effet un fragile équilibre sur lequel reposait un humanisme qui se voulait scientifique et objectif tout en plaçant l'homme moral avec sa personne juridique et éthique au sommet de la pyramide comme fin dernière de la nature. Mais cette disparition du projet, et finalement de l'âme du monde, n'a pas que des inconvénients. Au contraire même, d'un certain point de vue, il est heureux qu'il en soit ainsi ; que rien ne nous force à accepter l'idée d'un projet dans l'univers, car si le monde avait un projet objectif, nous n'aurions plus qu'a nous y soumettre. En fait, cela nierait pratiquement la possibilité de véritable innovation et de créativité, non seulement en général dans la nature, mais de créativité humaine en particulier. Nous serions inéluctablement entrainés dans une forme de totalitarisme du sens de l'univers, ou du sens de l'histoire, auquel chacun devrait se soumettre sous peine d'être excommunié comme hérétique, comme traître, ou comme fou.

Par ailleurs, tout cela n'empêche pas que nous sommes capables de faire des projets et que nous en faisons tous les jours, à quelqu'échelle que ce soit, individuel ou collectif, pour dans une heure, dans un an, 10 ans, un siècle et même l'éternité. Bien sûr, nous savons que le propre des projets est que leur réalisation est incertaine. Mais nous savons aussi que notre existence dans le temps ne peut pas s'en passer.

Bien sûr, la connaissance scientifique nous pousse à qualifier d'illusion notre croyance en la réalité objective déterminante de nos projets et surtout en leur efficacité, puisque nous apprenons qu'à travers nous-mêmes, notre comportement, nos sentiments, nos pensées (y compris notre croyance en ce que nous savons sur cette illusion), ce sont les interactions moléculaires de la chimie du système nerveux qui sont réellement déterminantes. Mais qualifier cette croyance d'illusion n'empêche personne, même parmi les biologistes réductionnistes les plus extrêmes, de faire des projets et d'essayer de les 
réaliser. Cela veut dire évidemment que l'opposition ne se situe pas au même niveau : notre capacité de faire des projets fait partie de notre expérience intuitive du réel pour laquelle la méthode scientifique est très mal adaptée ; tout comme notre expérience esthétique, et aussi notre capacité à porter des jugements éthiques, de l'ordre du bien et du mal et de la responsabilité personnelle, tous domaines pour lesquels la méthode scientifique est mal adaptée.

Quand nous prenons conscience de cela, de cette séparation nécessaire des domaines de légitimité, alors, la vision d'un monde sans projet, qui nous parle d'un langage de signes qui n'est pas celui que nous parlons pour dire ce que nous sentons et voulons dans notre vie de tous les jours, de ce monde ou l'homme n'est plus la mesure de toute chose, cette vision-là devient alors non pas une source de désespoir, mais au contraire de soulagement : nous sommes libérés seulement maintenant et grâce à cela, de ce que Nietzsche appelait le pouvoir des prêtres.

En effet, le monde qui parlait autrefois, celui qui était tout plein du projet d'un Dieu ou de la Nature, ne parlait jamais directement et simplement : le projet n'était jamais directement évident à tout le monde. Les voies de Dieu ont toujours été impénétrables... sauf à ceux qui savent déchiffrer le langage de la nature, entendre les appels, et indiquer aux autres quel est le vrai sens de leur existence et de leur histoire, au besoin par la force si c'est nécessaire.

Le pouvoir des prêtres a été dénoncé d'abord au nom de la raison et des lumières. Mais c'était une ruse. Car très vite, il est apparu que l'enjeu était en fait le remplacement des prêtres des anciennes religions par de nouveaux prêtres, ceux de la nouvelle religion, celle de la raison.

Et il a fallu longtemps, trois siècles, pour que la pratique scientifique se dégage de cette tentation globalisante de foumir une vision du monde totale, une théorie unifiée susceptible de résoudre tous les problèmes de l'humanité, y compris ses problèmes éthiques et d'organisation sociale et politique.

Cette tentation resurgit régulièrement, même chez des hommes de science qui ont du mal à y résister, et cèdent au besoin de dire « au nom de la science » ce qu'il faut faire, ce qui est bien et ce qui est mal.

Alors, dira-t-on, où chercher les critères ? Où chercher l'inspiration et la justification pour nos projets?

Et bien, justement dans l'inspiration et l'imagination qui n'ont pas besoin d'autre justification que rhétorique. Nous retrouvons là une distinction que les anciens connaissaient bien, entre les vertus de la raison et celles de l'imagination ; vertus respectives qui peuvent éventuellement se compléter, mais qu'il ne s'agit pas de confondre en sachant bien quelle est la place de chacune.

Ce sont l'imagination, l'intuition, l'inspiration qui fournissent leur contenu à nos projets et aujourd'hui, on ne peut plus sérieusement faire appel à rien d'autre. Le rôle de la raison est de les contrôler.

Il est important de ne pas mélanger les rôles ; que l'imagination source de projets, et la raison source de garde-fous et d'une certaine forme de vérité, 
n'intervertissent pas leurs rôles. Il existe une tradition, déjà chez Platon (Menon), qui attribue des qualités d'imagination aux prophètes et aux hommes politiques, et de raison aux hommes de science et aux philosophes. Il est remarquable que des philosophes comme Maimonide et Spinoza, qui s'opposent sur tellement de points, s'accordent pourtant sur cette répartition et sur l'association, surprenante à première vue, des hommes politiques aux prophètes. À la réflexion, cette association est naturelle et n'a rien d'étrange pour qui considère le discours prophétique comme une source très relative de vérité, devant toujours être contrôlée par la sagesse et la connaissance. Le Talmud, qui a nourri ces deux philosophes, exprime cette conception de façon explicite : «Le sage l'emporte sur le prophète ${ }^{9}$ ». La raison critique du sage et sa connaissance de la loi lui permettent de limiter et contrôler l'au-delà de la raison pourtant nécessaire que réalisent la prophétie et le projet politique.

Mais c'est là que les choses dérapent à nouveau. Cette situation d'incertitude est souvent difficile à supporter. Elle est, elle aussi, instable car elle crée une demande, une nostalgie ; celle de guides qui sauraient de façon garantie, quel est le vrai sens de l'aventure humaine. Et comme les hommes de science refusent en général désormais de jouer ce rôle de prêtres, alors on est prêt pour de nouveaux prêtres, ceux qui, utilisant le jargon scientifique, l'extrapolent dans tous les sens, pour pallier le manque de sens. C'est le retour d'une nouvelle alchimie, d'une astrologie dite scientifique, parfois aussi d'une mystique de l'écologie, qui vient ainsi foumir les nouveaux déchiffreurs d'avenir et du sens de l'histoire.

C'est la même nostalgie qui explique le retour beaucoup plus simple et radical à un nouvel obscurantisme religieux. Celui-ci tire sa force du constat d'échec d'une idéologie des Lumières qui croyait résoudre tous les problèmes de l'humanité grâce aux progrès de la rationalité scientifique. Ce nouvel obscurantisme tire aussi sa force de la critique de la technoscience dite occidentale - qui crée sans cesse de nouveaux dangers sans être capable d'apporter par elle-même de solutions aux problèmes d'ordre éthique, social et politique qu'elle crée de cette façon. Au contraire, le besoin d'une éthique qui ait en même temps la garantie d'être vraie, motive la recherche d'un intégrisme religieux, ou simplement idéologique, qui se veut à la fois scientifique et antimoderniste, ou une science nouvelle, "post-moderne ", ou "politiquement correcte », censurée et purifiée se confondrait avec la vérité d'une révélation ancienne.

Autrement dit, nous en sommes maintenant à un stade intéressant dans l'histoire de ceux qui prétendent entendre le monde parler et nous dévoiler son projet : nous sommes revenus au temps des oracles et des prophètes.

- Dans une première étape : les prêtres avaient perdu le pouvoir d'imposer la voie à suivre au nom d'une doctrine qui était vraie, parce que conforme à la vraie religion.

9. Talmud de Babylone, BabaBatra, 12a. 
- Dans une seconde étape : les hommes de science ont failli récupérer ce pouvoir au nom cette fois de doctrines vraies, parce que scientifiques. Mais ils y réussissent de moins en moins, de par le génie propre de la méthode scientifique qui est capable de se critiquer elle-même et de situer ses propres limites.

- Troisième étape : le temps est maintenant mûr pour le retour des prophètes, qui peuvent avoir parfois une certaine utilité, celle de suggérer des projets justement, mais à condition de savoir que leur discours, loin d'être celui de la vérité, est celui au mieux de la confusion, au pire de l'erreur et du mensonge.

Le domaine de la médecine nous foumit un exemple privilégié où cette situation permet d'expliquer un rapport à la science et à la technique qui semble au premier abord assez paradoxal : c'est aujourd'hui, maintenant que la médecine devient de plus en plus efficace, parce que de plus en plus scientifique, de plus en plus influencée par la biologie physico-chimique, mécanique et moléculaire, elle-même de plus en plus performante dans ses applications, c'est maintenant que cette médecine provoque la peur, le désenchantement et la nostalgie.

Il me semble que la raison de ce paradoxe est la suivante :

La subjectivité de la personne dans l'intimité de sa vie intérieure, et aussi la réalité sociale et juridique des rapports entre personnes responsables, sont totalement en dehors du champ de la biologie et à la limite même, niées par celle-ci. Même si l'expérience du praticien refuse une telle négation, dans la mesure ou cette expérience s'inscrit elle-même comme relation médecin-malade dans le champ social des rapports interpersonnels, elle ne trouve dans la science médicale comme science, aucun support. La science et la technique permettent à la médecine d'être efficace dans l'établissement de diagnostics et la mise en route de traitements par des méthodes objectives de plus en plus mécanisées et efficaces pour cela, mais au prix de l'élimination des sujets en cause. Et même si l'on veut tenir compte de ces sujets, soigner l'homme comme on dit et pas seulement la maladie, alors cela ne peut être que quelque chose en plus, plaque d'une certaine façon, ou le souci de la personne est comme un épiphénomène à propos duquel la biologie n'a rien à dire. Les sciences de l'homme ne sont pas d'un grand secours non plus car elles sont, elles aussi, dissociées du biologique et au mieux plaquées sur lui. En tout état de cause, elles sont loin d'avoir acquis la rigueur et l'efficacité des sciences biologiques et souffrent pour cela d'une espèce de complexe d'infériorité ou la biologie est soit divinisée, soit diabolisée.

Autrement dit, plus la médecine est scientifique, plus elle risque d'être dépersonnalisée. Et c'est cela qui explique d'abord la peur en même temps que la fascination. Il y a tentation à la fois de diviniser et de diaboliser son 
pouvoir et son efficacité, comme de façon générale le pouvoir des technosciences.

D'où le désenchantement et la peur. D'où aussi la nostalgie pour des médecines non scientifiques, dites douces ou parallèles, parées de toutes les vertus d'humanité, parfois à juste titre d'ailleurs, mais pas toujours non plus.

À ce propos, une mise au point est nécessaire, en particulier après certaines émissions de télévision ou l'on nous montre des témoignages sur l'efficacité, par exemple, de l'astrologie en médecine. Il est important de réaliser que la disparition de douleurs, d'angoisses ou d'autres symptômes, et même la guérison d'une maladie, n'est la preuve de rien du tout. Car n'importe quoi peut guérir sans que l'on sache toujours comment cela se produit.

Il existe des dizaines de techniques médicales dites parallèles, utilisées soit par des médecins, soit par des non-médecins, guérisseurs, chamans, marabouts, etc. Par ordre alphabétique, on peut citer : acupuncture, aromathérapie, astrologie, bioénergie, digitopuncture, fangothérapie, homéopathie, hydrothérapie, hypnose, ionisations, iridologie, magnétisme animal, méditation transcendantale, phytothérapie, régimes alimentaires divers, sophrologie, thalassothérapie, ultrasons, yoga, zen, etc. J'en oublie et j'en ignore certainement. Il ne s'agit pas de contester l'efficacité de tout cela. Bien au contraire. Tout marche. À des degrés divers bien sûr. Mais tout marche.

Et pour mieux s'en rendre compte, on peut observer que toutes ces techniques, bien que différentes, ont un certain nombre de points en commun. Elles ont d'abord en commun une critique de la médecine officielle, de son caractère dépersonnalisé, inhumain, etc., dont on a vu l'origine en partie justifiée.

D'autre part, chacune de ces techniques est accompagnée d'une théorie plus ou moins élaborée qui lui sert de fondement, car on ne peut pas dire ni même imaginer sincèrement qu' on fait n'importe quoi. Suivant les cas, cette théorie peut être très élaborée et très sophistiquée, comme l'astrologie dite scientifique, ou comme l'utilisation de machines censées capter et émettre des ondes plus ou moins bizarres inconnues de la physique, ou des théories sur des échanges d'énergie mâle ou femelle inspirées du Tao et du I King qui jouent sur l'ambiguïté de la notion d'énergie qui n'a rien à voir ici avec l'énergie physique, etc. Dans d'autres cas, la théorie utilisée est beaucoup moins élaborée et à la limite même, certains guérisseurs disent ne pas savoir comment ça marche : ils se sont découvert un don que certains expriment parfois comme un magnétisme - tout à fait mystérieux bien sur et qui n'a rien à voir avec le magnétisme mesurable des aimants et de la physique.

Quoiqu'il en soit, à l'origine de telle ou telle pratique de médecine parallèle, il existe un corpus théorique qui se présente comme un savoir soit traditionnel, soit scientifique, mais différent et non reconnu par la science officielle parce que celle-ci serait toujours aveugle et conservatrice et que les 
grandes découvertes seraient toujours méconnues et rejetées pendant longtemps avant d'être enfin acceptées.

Enfin, dernier caractère commun à toutes ces techniques de guérison et c'est évidemment le plus important — , chacune d'elles est capable d'amener, comme preuve de sa valeur et de son efficacité, des dizaines et des dizaines de témoignages de personnes qui tiennent à peu près, en toute sincérité, le même discours : « J'avais telle ou telle maladie, tel ou tel symptôme ; la médecine officielle n'a rien pu faire, ou seulement de façon très partielle et imparfaite. J'ai tout essayé jusqu'à ce que je rencontre $\mathbf{M}$. ou Mme X qui utilise cette technique Y. Et c'est seulement cela qui m'a soulagé. »

Ces témoignages sont sûrement véridiques, dans l'immense majorité des cas, mais là n'est pas la question. Car guérir une maladie ou soulager des douleurs n'est en aucune façon la preuve de la vérité du corpus théorique qui sert de fondement à la technique utilisée. Le nombre de paramètres qui intervient dans la survenue d'une maladie chez quelqu'un, dans sa singularité et dans son évolution vers le pis ou le mieux, est tellement grand que n'importe quoi peut guérir sans que l'on sache nécessairement comment cela s'est produit. La meilleure illustration en est évidemment l'efficacité de l'effet placebo ou, en toute connaissance de cause, on utilise un n'importe quoi dont on sait a priori qu'il n'a aucune raison d'être efficace. C'est cela qui explique la multiplicité des médecines parallèles et leur efficacité relative alors même qu'elles reposent chacune sur une théorie différente. On comprend bien que les succès thérapeutiques qu'elles peuvent avoir à leur actif ne sont en aucune façon la preuve de la vérité de telle ou telle théorie ou savoir sur lequel elles s'appuient et par lequel elles se définissent.

Grâce à la psychologie, et à la psychanalyse, avec par exemple la prise de conscience des phénomènes de transfert, et aussi de phénomènes psychosomatiques que l'on comprend encore mal, on commence à se faire certaines idées sur les raisons qui expliquent peut-être cet état de choses.

Quoi qu'il en soit, dans tous les cas encore une fois, le succès thérapeutique tout seul ne peut pas être la preuve de quoi que ce soit. Car la relation thérapeutique est trop indéterminée à cause du trop grand nombre de paramètres en jeu. Heureusement pour nous, la nature est bonne fille et il peut arriver que pratiquement n'importe quoi soit efficace sans que l'on sache exactement pourquoi. Et c'est cela qui laisse le champ libre à toutes interprétations et à toutes théorisations plus ou moins farfelues.

On pourrait objecter que cette observation s'applique aussi à la médecine officielle. Et en effet, s'il n'y avait comme soutien aux théories physiques et biologiques sur lesquelles elle s'appuie qu'un certain nombre de guérisons, on pourrait en dire exactement la même chose. Il s'agit la d'un cas particulier de ce que l'on appelle la sous-détermination des théories par les faits ${ }^{10}$. Quand on analyse le comportement d'un système constitué par un certain nombre — pas

10. Henri Atlan, Tout, non, peut-être, Seuil, Paris, 1991, p. 130-141 et p. $161-164$. 
forcément très élevé - de processus couples (tels que des réactions chimiques dans une cellule, des sécrétions endocriniennes dans un système de régulations hormonales, des intéractions entre populations de lymphocytes dans le système immunitaire, des réseaux de neurones dans le système nerveux, etc.), on se trouve en général dans une situation ou un grand nombre de modèles différents rendent compte également des faits d'observation. Autrement dit, nous souffrons non pas d'un défaut de théories, mais d'un excès de " bonnes " théories équivalentes bien que différentes, que l'on peut extrapoler de différentes façons, sans que nous ayons le moyen de déterminer laquelle serait plus "vraie" que les autres. Cela vient de ce que les faits d'observation qui nous sont accessibles sur un système in vivo dans son comportement intégré, sous la forme d'états stables de ce système, sont en général en nombre beaucoup plus petit que le nombre de modèles d'intéractions que l'on peut concevoir. En effet, le nombre d'états d'un système varie comme une puissance $\mathrm{N}$ du nombre d'états de chaque élément (ou $\mathrm{N}$ est le nombre d'éléments ou de processus couples qui constituent le système), tandis que le nombre de modèles $\mathrm{d}^{\prime}$ interconnexions varie comme une puissance $\mathrm{N}^{2}$ du nombre de valeurs possibles pour les différentes connexions. Il en résulte que plus $\mathbf{N}$ est grand, plus sont difficiles à réunir les conditions expérimentales ou le nombre d'états observables serait assez grand pour permettre d'éliminer le plus grand nombre possible de théories, idéalement toutes sauf une. C'est pourquoi, quand le système observé est un organisme dans son intégralité, dont les états sont définis de façon globale par l'existence de symptômes en nombre forcement très limités, cette sous-détermination des théories par les faits est énorme. Et la guérison, c'est-à-dire le passage d'un état avec certains symptômes à un état sans ces symptômes est très largement insuffisante pour permettre de trancher entre un grand nombre de théories différentes. Ceci s'applique à toute technique thérapeutique dont la seule validation possible est l'observation de son efficacité dans un certain pourcentage de cas. Mais le pari de la médecine scientifique, avec ses inconvénients et ses avantages, est que le savoir théorique sur lequel elle s'appuie est établi par ailleurs, en dehors de la thérapeutique, par les méthodes expérimentales de la biologie et de la physiopathologie, et non pas en sautant les étapes, par une efficacité thérapeutique directement observé sur des malades.

Enfin, à propos de l'argument sur les innovations scientifiques non reconnues, s'il est vrai qu'il est arrivé souvent que des idées nouvelles, considérées comme farfelues par la communauté scientifique, aient été ensuite reconnues comme valables, cela ne veut pas dire que réciproquement il suffit qu'une idée soit farfelue pour qu'elle soit vraie.

En conclusion, il est indispensable d'échapper à la tentation, soit de diviniser, soit de diaboliser la science. Et pour cela, il est certainement important de ménager un espace qui échappe à la toute-puissance de la technoscience, qu'il s'agisse de la vie intérieure ou de l'esthétique, ou de l'affrontement avec des problèmes éthiques ou politiques ou même de santé 
mentale et physique. La richesse des mythologies comme sources de sens possibles bien que multiformes, métaphoriques, et toujours destinés à être recrées par l'interprétation, est un élément probablement irremplaçable dans la constitution de cet espace. Mais il n'est pas question cependant de rejeter les apports de la science à la pensée critique ou de la technique à l'organisation de notre vie quotidienne. C'est alors qu'il faut éviter de se laisser enfermer dans la recherche d'une nouvelle idéologie unificatrice, disant à la fois ce qui est vrai et ce qu'il faut faire, dans une grande synthèse scientifico-mystique. Autrement dit, pour ne pas nous laisser étouffer par l'ordre de la technoscience, il n'est pas question que nous retournions au lavoir, à la bougie et aux sorciers guérisseurs, ni aux gourous du New Age ou des nouvelles anciennes religions. Comme on l'a vu, il ne s'agit pas, au nom d'une médecine hypertechnicienne, de nier la réalité de l'aide que peuvent parfois apporter des guérisseurs : les lavoirs lavent, les bougies éclairent, et les guérisseurs guérissent probablement dans la même proportion par rapport à la médecine moderne que les lavoirs par rapport aux machines à laver ou les bougies par rapport à la lumière électrique.

Et dans certaines circonstances, réclamant une certaine intimité, il peut être plus approprié d'organiser un éclairage à la bougie que de braquer de puissants spots électriques. Mais cela ne veut pas dire pour autant que nous verrons plus clair, même à l'intérieur de nous-mêmes, en renonçant à l'usage de l'électricité ou à l'usage de l'esprit critique que nous apprend la recherche scientifique, avec - et grâce à - ses limitations.

Hôtel-Dieu de Paris

Hôpital Hassidique de Jérusalem 